\title{
Efeito da atividade física associada à orientação alimentar em adolescentes obesos: comparação entre o exercício aeróbio e anaeróbio
}

\author{
Renata Viccari Sabia1, José Ernesto dos Santos² e Rosane Pilot Pessa Ribeiro ${ }^{3}$
}

\section{RESUMO}

A obesidade na adolescência é uma doença universal de prevalência mundial crescente, assumindo caráter epidemiológico como problema de saúde pública na sociedade moderna. Este trabalho teve como objetivo comparar o efeito do exercício físico aeróbio contínuo e anaeróbio intermitente associado à orientação alimentar sobre a composição corporal, as medidas bioquímicas e a capacidade física de adolescentes obesos. Foram investigados 28 adolescentes (média de idade = 13 anos), com índice de massa corporal (IMC) acima do percentil 95 para a idade e sexo, distribuídos em dois grupos de forma aleatória: exercício de caminhada contínua (GEC; $n=13$ ) e exercício de corrida intermitente (GEl; $n=15$ ) e submetidos a um programa de treinamento físico três vezes por semana durante 16 semanas por 20 a 40 minutos. A atividade de orientação nutricional ocorreu uma vez por semana, em grupo, por 60 minutos, durante todo o experimento. Foram realizadas, no período inicial e final, medidas de peso e altura, pregas subcutâneas, circunferências do braço (CB) e muscular do braço (CMB), composição corporal por bioimpedância elétrica, análises bioquímicas séricas (glicemia e lipídios) e determinação direta de consumo máximo de oxigênio ( $\mathrm{V}_{2}$ max) e limiar anaeróbio (LAn). Como resultados antropométricos, observamos diminuição do IMC e das pregas cutâneas com diferença significativa nos dois grupos (GEC e GEI). Na avaliação bioquímica, houve diminuição significativa nos níveis séricos de HDL e LDL, colesterol total, e aumento do triglicérides e glicemia no GEC, embora mantendo-se dentro dos valores de normalidade. No GEl, o HDL e triglicérides tiveram diminuição significativa. $\mathrm{O} \mathrm{V}_{2}$ max aumentou estatisticamente nos dois grupos. Concluímos que a atividade física proposta e a orientação alimentar, tanto para o GEC quanto para GEI, foi suficiente e satisfatória, promovendo diminuição ponderal, melhora da composição corporal, dos níveis lipídicos e aumento na capacidade aeróbia dos adolescentes.

\section{RESUMEN}

\section{Efecto de la actividad física asociada a la orientación alimen- tar en adolescentes obesos: comparación entre el ejercicio aeróbico y anaeróbico}

La obesidad en la adolescencia es una enfermedad universal de prevalencia mundial creciente, asumiendo un carácter epidemioló-

1. Educadora Física, Mestre em Saúde Pública pela Escola de Enfermagem de Ribeirão Preto-USP.

2. Médico, Prof. Associado do Departamento de Clínica Médica da Faculdade de Medicina de Ribeirão Preto-USP.

3. Nutricionista, Prof ${ }^{a}$ Doutora do Departamento de Enfermagem Materno-Infantil e Saúde Pública da Escola de Enfermagem de Ribeirão Preto-USP.

Recebido em 3/10/03. 2a versão recebida em 10/2/04. Aceito em 24/6/04.

Endereço para correspondência: Renata Viccari Sabia, Rua Jurumirim, 1.060 - 86870-000 - Ivaiporã, PR. Tel.: (43) 472-3913. E-mail: rvsabia@ig. com.br
Palavras-chave: Obesidade. Adolescência. Exercício aeróbio e anaeróbio. Palabras-clave: Obesidad. Adolescencia. Ejercicio aerobio y anaerobio.

gico como problema de salud pública en la sociedad moderna. Este trabajo tiene como objetivo comparar el efecto del ejercicio físico aeróbico continuo y anaeróbico intermitente asociado a la orientación alimentar respecto a la composición corporal, a las medidas bioquímicas y a la capacidad física de adolescentes obesos. Se investigaron 28 adolescentes (promedio de edad = 13 años), con indice de masa corporal (IMC) arriba del percentil 95 para la edad y el sexo, los cuales se distribuyeron en dos grupos en forma aleatoria: ejercicio de caminata continua (GEC; $n=13$ ) y ejercicio de carrera intermitente (GEl; $n=15)$. Los grupos también se sometieron a un programa de entrenamiento físico tres veces por semana durante 16 semanas por 20 a 40 minutos. La actividad de orientación nutricional sucedió una vez por semana, en grupo, por 60 minutos, durante todo el experimento. Se realizaron, en el periodo inicial y final, mediciones de peso y altura, pliegas subcutáneas, circunferencias del brazo (CB) y muscular del brazo (CMB), composición corporal por bioimpedancia eléctrica, análises bioquímicos séricos (glicemia y lipidios) y determinación directa del consumo máximo de oxígeno ( $\dot{\mathrm{V}} \mathrm{O}_{2} \mathrm{max}$ ) y límite anaerobio (LAn). Como resultados antropométricos, observamos una reducción del IMC y de las pliegas subcutáneas, con diferencia significativa en los dos grupos (GEC e GEI). En la evaluación bioquímica, hubo una disminución significativa de los niveles séricos de HDL e LDL, colesterol total y aumento de triglicéridos y glicemia en el GEC, aunque se hayan mantenido de acuerdo a los valores de normalidad. En el GEl, el HDL y triglicéridos sufrieron una reducción significativa. El $\dot{V} \mathrm{O}_{2}$ max aumentó estadísticamente en los dos grupos. Concluimos que la actividad física sugerida y la orientación alimentar, tanto para el GEC como para GEI, fue suficiente y satisfactoria y produjo una disminución ponderal, mejora de la composición corporal, de los niveles lipídicos y aumento de la capacidad aerobia de los adolescentes.

\section{INTRODUÇÃO}

A obesidade é considerada doença universal de prevalência crescente e hoje assume caráter epidemiológico, como o principal problema de saúde pública na sociedade moderna ${ }^{(1)}$. O indivíduo é considerado obeso quando a quantidade de gordura relativa à massa corporal se iguala ou excede a $30 \%$ em mulheres e a $25 \%$ em homens e a obesidade grave é caracterizada por um conteúdo de gordura corporal que exceda $40 \%$ em mulheres e $35 \%$ em homens ${ }^{(2)}$.

Devido ao fato de o índice de massa corporal (IMC) evidenciar uma proporção entre estatura e massa corporal, não quantificando a gordura corporal, a World Health Organization ${ }^{(3)}$ não utiliza mais o termo obesidade e, sim, sobrepeso I (IMC entre 25 e 29,9kg/m²), 
sobrepeso II (IMC entre 30 e 39,9kg/m²) e sobrepeso III (IMC maior ou igual a $40 \mathrm{~kg} / \mathrm{m}^{2}$.

A obesidade na infância e na adolescência está-se tornando problema cada vez mais freqüente, estando relacionada a diversas doenças, com aumento do risco de morbidade e mortalidade. Nos países desenvolvidos como os Estados Unidos, é considerado importante problema de saúde pública(4).

Sabemos que existem diferentes causas que desenvolvem a obesidade, entre eles, os aspectos bioquímicos, genéticos, fatores psicológicos, fatores fisiológicos e fatores ambientais(5).

A manutenção estável do peso e composição corporal durante os anos resulta de um balanço preciso entre a ingestão e o gasto energético(6); um desequilíbrio nesta relação desencadeia o processo da obesidade ${ }^{(7-9)}$. Sendo assim, a falta de atividade física e os distúrbios alimentares são hoje, sem dúvida, os principais fatores predisponentes para a obesidade $(6,10,11)$.

A transmissão familiar da obesidade é bem conhecida. No entanto, membros de uma mesma família estão expostos a hábitos culturais e dietéticos que acabam influenciando, sobremaneira, o ganho de massa corporal. Isso evidencia que, além da herança genética, a influência ambiental também acaba desempenhando papel importante no desenvolvimento da obesidade ${ }^{(12)}$.

A redução da ingestão de alimentos preparados em casa, substituídos por alimentos industrializados (fast-foods, salgadinhos e doces), o aumento do consumo de refrigerantes e de grande proporção de calorias derivadas de gordura e a redução de atividade física são fatores etiológicos responsáveis pelo crescimento da prevalência da obesidade nas populações urbanas do ocidente ${ }^{(12,13)}$

De acordo com Fisberg(8), a obesidade na infância tem como importância a possibilidade de sua manutenção na vida adulta. Se nas idades menores a morbidade não é freqüente, no adulto a situação é de risco e leva a aumento da mortalidade, por associação com a doença arteriosclerótica, hipertensão e alterações metabólicas. No adolescente, somam-se a isso todas as alterações do período de transição para a idade adulta, a baixa auto-estima, o sedentarismo, lanches malbalanceados em excesso e a enorme suscetibilidade à propaganda consumista.

Diante disso, conclui-se que, por ser a obesidade doença de difícil tratamento na vida adulta, sua prevenção, evitando surgimento já na infância ou adolescência, e seu tratamento, impedindo a evolução dos casos já diagnosticados, são de fundamental importância, melhorando o prognóstico destes pacientes na idade adulta.

Dessa forma, promover o aumento da atividade física e incentivo à aquisição de hábitos alimentares saudáveis, criando condições objetivas para sua realização, seriam, provavelmente, os principais componentes de políticas de uma vida saudável entre adolescentes.

A obesidade infantil e na adolescência requer, para seu tratamento, uma equipe multidisciplinar, agregando médicos pediatras e endocrinologistas, psicólogos, nutricionistas e fisiologistas do exercício(8,14).

Para Pollock e Wilmore(1), a motivação e a responsabilidade do sujeito consigo mesmo são fatores importantes no tratamento da obesidade.

Quando se maneja a alimentação de uma criança, deve-se ter em mente que se está diante de um organismo em crescimento, com necessidade energética de vitaminas, sais minerais, proteínas e fibras; deve-se então valorizar essas necessidades elaborando uma alimentação equilibrada e individualizada(14)

Utilizado como umas das estratégias na prevenção da obesidade na infância e adolescência, o exercício físico tem papel adjuvante no tratamento da obesidade, pois o gasto calórico proveniente da atividade física se mostra como grande aliado na perda de massa corporal, sendo também fator contribuinte para aumento nos níveis de aptidão física(1). O exercício físico especialmente na resistência muscular localizada, aumento da capacidade aeróbia, diminuição da massa corporal e percentagem de gordura corporal, sem, no entanto, limitar a velocidade de crescimento linear ou reduzir a massa corporal magra(15).

Diante dessas considerações e a partir da revisão bibliográfica realizada, observamos a existência de vários estudos na literatura que comprovam o efeito positivo da atividade física sobre a redução do tecido adiposo. Entretanto, estes consideram apenas a duração do trabalho realizado, sendo que poucos demonstram a interferência do tipo de exercício, quanto à predominância do metabolismo energético empregado, aeróbio ou anaeróbio, durante o movimento.

Sendo assim, acreditamos que investigar aspectos do exercício físico aeróbio e anaeróbio irá contribuir para melhor entendimento desses mecanismos, produzindo conhecimentos específicos que podem favorecer melhores resultados no tratamento da obesidade.

O objetivo deste trabalho foi comparar o efeito do exercício físico aeróbio contínuo e anaeróbio intermitente associado à orientação alimentar em adolescentes obesos sobre a redução da massa corporal e composição corporal, lipídios sanguíneos e capacidade de trabalho físico.

\section{MÉTODOS}

Os voluntários foram recrutados por meio de anúncios veiculados nos meios de comunicação (jornal da USP e televisão de Ribeirão Preto-SP).

Os critérios de inclusão considerados foram: idade de 12 a 14 anos, IMC acima do percentil 95(16), considerado obeso para a idade, e sexo e disponibilidade total para participar das atividades de educação física (três vezes/semana, das 14:00 as 15:15 horas) e orientação nutricional (uma vez/semana, por 1,5 hora). Os sujeitos que não apresentavam essas características foram considerados excluídos.

Com os dados de peso e altura, calculou-se o índice de massa corporal (IMC), conhecido também como índice de Quetelet ${ }^{(17)}$, correspondendo à relação entre o peso em kg e o quadrado da estatura em metros. Para análise desse parâmetro, utilizou-se classificação proposta por Must et al..(16), considerando-se obesidade quando o percentil fosse igual ou maior que 95.

O cronograma de atividades constou de: avaliação inicial, antes das atividades de educação física e orientação nutricional, composta por: 1) medidas de composição corporal (peso, altura, pregas e circunferências, bioimpedância elétrica), avaliação bioquímica (dosagens séricas de colesterol e frações, triglicérides e glicose), determinação do consumo máximo e limiar anaeróbio; 2) avaliação final, após 16 semanas de atividade física e orientação nutricional, constando dos mesmos exames e medidas tomadas no inicio. As avaliações foram realizadas de forma cega, ou seja, o avaliador não sabia em qual dos grupos o voluntário estava alocado.

Os pais ou responsáveis pelos adolescentes assinaram termo de consentimento livre e esclarecido antes do início do protocolo experimental, tendo este projeto sido anteriormente aprovado pelo Comitê de Ética em Pesquisa da Escola de Enfermagem de Ribeirão Preto-USP (no 0157/2001).

Para assegurar que os mesmos não apresentavam contra-indicações para o exercício físico, foi solicitado um atestado médico antes do inicio da pesquisa.

Para medida de peso utilizou-se uma balança de plataforma da marca Filizola, com carga máxima de $150 \mathrm{~kg}$ e divisões de $100 \mathrm{~g}$. A balança foi aferida antes de cada medição e os voluntários foram pesados em pé, descalços e usando roupas leves (short, bermuda, camiseta de manga curta ou sem manga).

A medida da estatura foi verificada na balança de aferição do peso corporal, utilizando a haste de metal inflexível marcada de $\mathrm{cm} / \mathrm{cm}$ até a altura de $2 \mathrm{~m}$, posicionado o sujeito descalço, encostando os calcanhares, quadris, escápula e parte occipital do crânio na superfície do aparelho. 
As dobras cutâneas (tríceps e subescapular) foram medidas com um compasso Plicômetro de Lange (escala de 0,60 milímetro, com precisão mais ou menos um milímetro), no lado não dominante do corpo, e para a circunferência braquial foi utilizada uma fita métrica não extensível. Três medidas foram realizadas e a média utilizada para análise.

Para a realização da bioimpedância elétrica, a resistência e a reactância corporal foram medidas utilizando-se um analisador de composição corporal (modelo BIA 101A, RJL Systems). Os participantes foram orientados a ficar de jejum desde as 24 horas do dia anterior, não realizar atividade física imediatamente antes do exame e no dia anterior, não ingerir bebida alcoólica.

As coletas de sangue $(4 \mathrm{ml})$ para análise bioquímica das lipoproteínas (HDL e LDL), triglicérides e colesterol foram realizadas no Laboratório de Nutrição do Hospital das Clínicas da Faculdade de Medicina de Ribeirão Preto/USP. Todos os adolescentes se encontravam em jejum de 12 horas. O método empregado foi a Dimensionxpand, equipamento Dade Behring. Para a análise de glicemia (5ml), realizada no Laboratório de Bioquímica do Hospital das Clínicas da Faculdade de Medicina de Ribeirão Preto/USP, utilizou-se como metodologia a hexoquinase, equipamento Dade Behring, modelo Dimension RXL.

Para identificar a intensidade de exercício (W) correspondente ao limiar anaeróbio e determinar o consumo máximo de oxigênio, os sujeitos foram submetidos a protocolos de exercício realizados em esteira rolante no laboratório LAFINE da Universidade de Ribeirão Preto - UNAERP.

$\checkmark$ Para determinação da intensidade correspondente ao limiar anaeróbio (LAn), planejou-se uma corrida contínua e progressiva realizada em esteira rolante (/mbramed - ATL 10200), com carga inicial de $6 \mathrm{~km} / \mathrm{h}$ e incremento de $1 \mathrm{~km} / \mathrm{h}$ a cada minuto até exaustão. Ao final de cada carga, foram mensuradas a freqüência cardíaca (FC) por meio de monitor de freqüência cardíaca (Polar-Vantage $\mathrm{XL}$ ) e amostras de 25 microlitros de sangue arterializado coletados do lóbulo da orelha, por meio de tubos capilares heparinizados e armazenados em tubos ependorfs contendo 50 microlitros de $\mathrm{NaF}$ $1 \%$. Posteriormente, estas amostras foram analisadas enzimaticamente (Yelowspring 2700 stat), a fim de se observar a concentração de lactato [lac] em cada intensidade. Dessa forma, para cada carga foram observadas a FC e a concentração de lactato e, por meio de interpolação linear, foi encontrada a intensidade (watts) e a FC correspondente a 3,5mM de lactato, limiar anaeróbio (Heck et al., 1985). A determinação da FC ao final de cada estágio dos testes progressivos foi efetuada pelo equipamento Polar Vantage $\mathrm{XL}$.

$\checkmark$ Para determinação do consumo máximo de oxigênio $\left(\dot{V} \mathrm{O}_{2} \max \right)$, foi mensurado continuamente durante todo o protocolo de avaliação, a partir do gás expirado, pelo sistema Vista CPX, Vacumed, 1996. Este sistema permite a dosagem do $\mathrm{O}_{2}$ e $\mathrm{CO}_{2}$ do ar expirado a cada 30 segundos, utilizando-se da câmara de mistura e analisadores Oxygen Analyser OM-11 e Carbon dioxide analiser LB-2, respectivamente. A determinação do volume de ar expirado foi obtido por meio de fluxômetro Flow transducer $\mathrm{K}-520$. Os sinais dos equipamentos foram devidamente processados e analisados por meio de software do sistema Vista CPX, a fim de nos fornecer os valores de consumo de oxigênio $\left(\dot{V}_{2}\right)$, produção de $\mathrm{CO}_{2}$, razão de trocas respiratórias $(\mathrm{R})$ e ventilação pulmonar (VE btps) a cada 30 segundos. Para considerarmos que durante o teste os indivíduos atingiram o $\mathrm{VO}_{2}$ max, foram adotados os critérios propostos por Shephard et al. (1968).

Após a realização da avaliação inicial, os voluntários foram subdivididos em dois grupos aleatórios; isto é: foi determinado o primeiro grupo experimental de acordo com um sorteio do tipo de exercício que este iria realizar. Sendo assim, o primeiro grupo realizou atividades de predominância aeróbia (GEC =13) e o segundo, de predominância anaeróbia $(G E I=15)$ e foram submetidos a um programa de treinamento físico, três vezes por semana durante 16 semanas, constando de caminhada contínua (GEC) e corrida intermitente (GEI).

A fim de proporcionar aos voluntários uma adaptação gradual ao estresse da atividade física nas primeiras e segundas semanas, a duração do trabalho foi, respectivamente, de 20 e $30 \mathrm{~min}$. A partir da terceira semana esse período foi de $40 \mathrm{~min}$, até o final do experimento.

O treinamento aeróbio (GEC) constou de um período de $10 \mathrm{mi}-$ nutos de aquecimento (calistenia e alongamento), uma parte principal de 20 a 40 minutos de caminhada contínua de baixa intensidade (predominância aeróbia) realizada na pista de atletismo, com carga de trabalho específica para cada voluntário; segundo os resultados da avaliação cardiorrespiratória $\left(\dot{\mathrm{VO}}_{2} \max \right)$, trabalhou-se com a intensidade de $80-85 \%$ do $\left(\mathrm{VO}_{2}\right.$ max), que foi monitorada pela freqüência cardíaca. No final, eles realizavam 15 minutos de esportes com bola (volei, basquete e futebol), como forma de motivação.

O treinamento anaeróbio (GEI) foi realizado utilizando-se o método do "treinamento intervalado"(18-20), caracterizado por atividade física com duração de 20 a 40 minutos, terminando com 60 minutos. O exercício proposto foi corrida intermitente de alta intensidade (predominância anaeróbica) e circuito com peso. Os circuitos eram intercalados por dia da semana com as corridas, trabaIhando apenas os grandes grupos musculares, também com intervalos de um minuto por exercício. A intensidade determinada foi de $95-105 \%$ do $\left(\dot{\mathrm{V}} \mathrm{O}_{2} \max \right)$, que foi monitorada pela freqüência cardíaca.

O treinamento de corrida intervalada foi realizado na pista de atletismo, onde, no primeiro mês, os voluntários completavam seis séries de 30 segundos, com intervalo de um minuto por série, percorrendo uma distância de 100 metros por série. Os voluntários terminaram o treinamento realizando 10 séries de 35 segundos, com um minuto de intervalo, percorrendo uma distância de 210 metros

As atividades de orientação nutricional foram desenvolvidas por um nutricionista no CEFER - USP-Ribeirão Preto com todos os adolescentes durante a pesquisa, antes das atividades esportivas, uma vez por semana em grupo, com duração programada de 60 minutos. Foi desenvolvido um programa de reeducação alimentar baseado em modificações de hábitos e comportamentos, diante da identificação de erros alimentares e incentivando a prática de uma alimentação saudável e equilibrada.

Os dados coletados foram organizados em um banco de dados, utilizando-se o programa SPSS (Statistical Packge for Social Sciences). Optamos por mostrar os resultados como mediana e amplitude semiquartil (Q1 e Q3), utilizando a análise estatística não-paramétrica (teste de Mann-Whitney e Wilcoxon, $p<0,05$ ), devido ao tamanho pequeno da amostra.

\section{RESULTADOS}

Comparando-se os valores iniciais das variáveis medidas entre os dois grupos, observou-se que o peso corporal, IMC e CB (circunferência braquial) não foram diferentes estatisticamente (teste de Mann-Whitney). Diante disso, os resultados a apresentar serão comparados dentro de cada um dos dois grupos separadamente, ou seja, a evolução do valor final em relação ao inicial. Para isso, foi realizada a análise estatística não-paramétrica (teste de Wilcoxon, $p<0,05)$, considerando o tamanho pequeno da amostra.

A tabela 1 mostra os dados relativos ao peso corporal total, altura e IMC nas fases pré e pós-treinamento. Para as medidas de peso corporal total, foi observada diminuição nos dois grupos, assim como aumento para as medidas de altura conforme tabela a seguir.

O efeito do treinamento na redução do IMC foi bastante positi$v o$, tendo diminuição com diferença estatística nos dois grupos ( $p$ $=0,001$ para o GEC e GEI) entre os valores iniciais e finais de cada grupo. 
TABELA 1

Peso corporal total, altura e índice de massa corporal (IMC) dos grupos aeróbio contínuo (GEC) e anaeróbio intermitente (GEI), pré e pós-treinamento. Valores expressos como mediana (Me) e amplitude semi-quartil (Q1 e Q3)

\begin{tabular}{|c|c|c|c|c|c|c|}
\hline \multirow[t]{2}{*}{ Grupo } & \multicolumn{2}{|c|}{ Peso total (kg) } & \multicolumn{2}{|c|}{ Altura (m) } & \multicolumn{2}{|c|}{ IMC $\left(\mathbf{k g} / \mathrm{m}^{2}\right)$} \\
\hline & Pré & Pós & Pré & Pós & Pré & Pós \\
\hline \multicolumn{7}{|c|}{ GEC $(n=13)$} \\
\hline $\mathrm{Me}$ & 80,6 & 80,3 & 1,64 & 1,66 & 30 & $28,6^{*}$ \\
\hline Q1 & 71,5 & 74,4 & 1,57 & 1,63 & 29 & 28 \\
\hline Q3 & 95 & 94 & 1,68 & 1,7 & 32,05 & 30,5 \\
\hline \multicolumn{7}{|c|}{ GEI $(n=16)$} \\
\hline $\mathrm{Me}$ & 87 & 81 & 1,65 & 1,68 & 31,3 & $29,4 *$ \\
\hline Q1 & 82,1 & 77 & 1,61 & 1,64 & 29,6 & 27,1 \\
\hline Q3 & 90,35 & 87 & 1,69 & 1,72 & 32,4 & 29,85 \\
\hline
\end{tabular}

* $p<0,05$ em relação ao pré-treinamento, teste de Wilcoxon

Para a variável CB (circunferência de braço), verificamos diminuição significativa dos valores finais em relação aos iniciais dentro dos dois grupos, mostrados na tabela 2 ( $p=0,005$ para o GEC e $\mathrm{p}=0,006$ para o GEl, teste de Wilcoxon). Já para CMB (circunferência muscular do braço), não observamos o mesmo efeito.

Em relação às medidas de dobras cutâneas representadas na tabela 3, podemos observar diminuição estatisticamente significativa na medida de PCT (prega cutânea tricipital), PCSE (prega cutânea subescapular) e PCSI (prega cutânea supra-ilíaca) no pós-treinamento em relação ao pré-exercício no GEC ( $p=0,003, p=0,008$, $p=0,04$, respectivamente) e no GEI ( $p=0,017, p=0,001, p=$ 0,03 , respectivamente)

TABELA 2

Medidas antropométricas (CB) circunferência de braço; (CMB) circunferência muscular do braço, para os grupos aeróbio contínuo (GEC) e anaeróbio intermitente (GEI), pré e pós-treinamento. Valores expressos como mediana (Me) e amplitude semiquartil (Q1 e Q3)

\begin{tabular}{|c|c|c|c|c|}
\hline \multirow[t]{2}{*}{ Grupo } & \multicolumn{2}{|c|}{$\mathrm{CB}(\mathrm{cm})$} & \multicolumn{2}{|c|}{ CMB (cm) } \\
\hline & Pré & Pós & Pré & Pós \\
\hline \multicolumn{5}{|c|}{ GEC $(n=13)$} \\
\hline $\mathrm{Me}$ & 35 & $34^{*}$ & 25,8 & 25,7 \\
\hline Q1 & 34 & 33 & 24,5 & 24,5 \\
\hline Q3 & 37,75 & 36 & 27,8 & 27,6 \\
\hline \multicolumn{5}{|c|}{$\mathrm{GEI}(\mathrm{n}=16)$} \\
\hline $\mathrm{Me}$ & 35 & $34^{*}$ & 23,9 & 23,6 \\
\hline Q1 & 34,25 & 33 & 23,05 & 22,25 \\
\hline $\mathrm{Q} 3$ & 36,75 & 36 & 25,4 & 24,8 \\
\hline
\end{tabular}

* $p<0,05$ em relação ao pré-treinamento, teste de Wilcoxon

TABELA 3

Dobras cutâneas dos grupos aeróbio contínuo (GEC) e anaeróbio intermitente (GEI), pré e pós-treinamento. Valores expressos como mediana ( $\mathrm{Me}$ ) e amplitude semiquartil (01 e 03 )

\begin{tabular}{|c|c|c|c|c|c|c|c|c|}
\hline \multirow[t]{2}{*}{ Grupo } & \multicolumn{2}{|c|}{ PCT (mm) } & \multicolumn{2}{|c|}{ РCB $(\mathrm{mm})$} & \multicolumn{2}{|c|}{ PCSE (mm) } & \multicolumn{2}{|c|}{ PCSI (mm) } \\
\hline & Pré & Pós & Pré & Pós & Pré & Pós & Pré & Pós \\
\hline \multicolumn{9}{|c|}{ GEC $(n=13)$} \\
\hline $\mathrm{Me}$ & 28,5 & $27^{*}$ & 23 & 21 & 27 & $25^{*}$ & 38 & $36^{*}$ \\
\hline Q1 & 25,75 & 25 & 20 & 18 & 26 & 25 & 37 & 32 \\
\hline Q3 & 30 & 28 & 25 & 25 & 32 & 31 & 43 & 41 \\
\hline \multicolumn{9}{|c|}{ GEI ( $n=16)$} \\
\hline $\mathrm{Me}$ & 36 & $35^{*}$ & 30 & 30 & 34 & $35^{*}$ & 50 & $48^{*}$ \\
\hline Q1 & 35 & 33,5 & 25 & 25 & 31 & 27 & 43,5 & 41 \\
\hline Q3 & 39 & 37 & 35 & 35 & 40 & 39 & 55,5 & 52,5 \\
\hline
\end{tabular}

* $p<0,05$ em relação ao pré-treinamento, teste de Wilcoxon

PCT = prega cutânea tricipital; $\mathrm{PCB}$ = prega cutânea bicipital; PCSE = prega cutânea subescapular; PCSI = prega cutânea supra-ilíaca
Quanto à composição corporal analisada por bioimpedância elétrica, depois do treinamento físico, houve diferença estatisticamente significativa nos dois grupos nas variáveis medidas. Na massa gorda, observamos diminuição no GEC ( $p=0,001)$ e no GEI ( $p=$ 0,015 ) e aumento da massa magra (GEC, $p=0,001$; $G E I, p=0,015$ ), e água corporal total (GEC e GEI, $p=0,001)$. Esses valores estão representados na tabela 4.

\begin{tabular}{|c|c|c|c|c|c|c|}
\hline \multicolumn{7}{|c|}{$\begin{array}{c}\text { TABELA } 4 \\
\text { Composição corporal (massa gorda, massa magra e } \mathrm{H}_{2} \mathrm{O} \text { ) para } \\
\text { os grupos aeróbio contínuo (GEC) e anaeróbio intermitente (GEI), } \\
\text { pré e pós-treinamento. Valores expressos como mediana (Me) } \\
\text { e amplitude semiquartil ( } \mathrm{Q1} \text { e Q3) }\end{array}$} \\
\hline \multirow[t]{2}{*}{ Grupo } & \multicolumn{2}{|c|}{ Massa gorda (\%) } & \multicolumn{2}{|c|}{ Massa magra (\%) } & \multicolumn{2}{|c|}{$\mathrm{H}_{2} \mathrm{O}(\%)$} \\
\hline & Pré & Pós & Pré & Pós & Pré & Pós \\
\hline \multicolumn{7}{|c|}{ GEC ( $n=13)$} \\
\hline $\mathrm{Me}$ & 34 & $32 *$ & 66 & $68^{*}$ & 48 & $50 *$ \\
\hline Q1 & 31 & 27 & 58 & 62 & 42 & 45 \\
\hline Q3 & 42 & 38 & 69 & 73 & 50 & 53 \\
\hline \multicolumn{7}{|c|}{ GEI $(n=16)$} \\
\hline Me & 28 & $25^{*}$ & 72 & $74^{*}$ & 53 & $55^{*}$ \\
\hline Q1 & 25 & 22,5 & 69 & 70,5 & 50,5 & 52 \\
\hline Q3 & 31 & 29,5 & 75 & 77,5 & 55 & 57 \\
\hline
\end{tabular}

* $p<0,05$ em relação ao pré-treinamento, teste de Wilcoxon

Quanto à avaliação bioquímica das concentrações plasmáticas de lipoproteínas HDL, LDL e de colesterol total dos grupos (GEC, GEI), os resultados estão apresentadas na tabela 5 .

Os valores iniciais das medidas analisadas entre os dois grupos foram diferentes estatisticamente (teste de Mann-Whitney) em relação aos níveis de triglicérides $(p=0,009)$ e glicemia $(p=0,027)$. Comparando os valores finais em relação aos iniciais dentro de cada grupo, notamos que no GEC houve diminuição significativa nas concentrações de HDL e LDL ( $p=0,002$, teste de Wilcoxon). Já no GEI, o HDL diminuiu estatisticamente $(p=0,002)$ enquanto o valor de LDL aumentou, sem diferença estatística. Vale ressaltar que a média inicial tanto do HDL quanto do LDL eram considerados dentro da normalidade (LDL $<110$; 110-129) HDL ( $\geq 35$ ).

No nível de colesterol total, ocorreu melhora significativa apenas no GEC ( $p=0,006$, teste de Wilcoxon) com diminuição do valor final em relação ao inicial.

\section{TABELA 5}

Medidas bioquímicas (HDL, LDL) e colesterol total dos grupos aeróbio contínuo (GEC) e anaeróbio intermitente (GEI), pré e pós-treinamento.

Valores expressos como mediana (Me) e amplitude semiquartil (01 e Q3)

\begin{tabular}{|c|c|c|c|c|c|c|}
\hline \multirow[t]{2}{*}{ Grupo } & \multicolumn{2}{|c|}{ HDL (mg/dl) } & \multicolumn{2}{|c|}{ LDL (mg/dl) } & \multicolumn{2}{|c|}{$\begin{array}{c}\text { Colesterol } \\
\text { total }(\mathrm{mg} / \mathrm{dl})\end{array}$} \\
\hline & Pré & Pós & Pré & Pós & Pré & Pós \\
\hline \multicolumn{7}{|c|}{ GEC $(n=13)$} \\
\hline $\mathrm{Me}$ & 42 & $35^{*}$ & 101 & $78^{*}$ & 169 & $145^{*}$ \\
\hline Q1 & 37 & 33 & 85 & 67 & 136 & 123 \\
\hline Q3 & 46 & 40 & 121 & 96 & 182 & 153 \\
\hline \multicolumn{7}{|c|}{ GEI $(n=16)$} \\
\hline $\mathrm{Me}$ & 41 & $38^{*}$ & 83 & 81,2 & 139 & 156 \\
\hline Q1 & 37,5 & 32,5 & 69,4 & 64,6 & 133,5 & 127 \\
\hline Q3 & 49 & 41,5 & 101,9 & 110,4 & 184,5 & 179 \\
\hline
\end{tabular}

* $p<0,05$ em relação ao pré-treinamento, teste de Wilcoxon

Em relação aos níveis de glicemia e triglicérides, como já citado anteriormente, os valores iniciais foram diferentes estatisticamente entre os grupos. Observando a evolução da concentração de 
glicemia no GEC, curiosamente, houve aumento significativo ( $p=$ 0,021, teste de Wilcoxon), efeito esse não observado no GEI. Houve, também, aumento significativo na resposta de triglicérides no GEC $(p=0,05)$ e diminuição no GEl $(p=0,03)$ assim representados na tabela 6.

\begin{tabular}{|c|c|c|c|c|}
\hline \multicolumn{5}{|c|}{$\begin{array}{c}\text { TABELA } 6 \\
\text { Medidas bioquímicas (TG) triglicérides e glicemia para os grupos } \\
\text { aeróbio contínuo (GEC) e anaeróbio intermitente (GEI), nas fases } \\
\text { de pré e pós-treinamento. Valores expressos como mediana } \\
\text { (Me) e amplitude semiquartil (Q1 e Q3) }\end{array}$} \\
\hline \multirow[t]{2}{*}{ Grupo } & \multicolumn{2}{|c|}{ TG (mg/dl) } & \multicolumn{2}{|c|}{ Glicemia (mg/dl) } \\
\hline & Pré & Pós & Pré & Pós \\
\hline \multicolumn{5}{|c|}{ GEC $(n=13)$} \\
\hline $\mathrm{Me}$ & 79 & $99 *$ & 93,3 & $99 *$ \\
\hline Q1 & 54 & 83 & 93,15 & 95 \\
\hline Q3 & 100 & 108 & 100,15 & 101 \\
\hline \multicolumn{5}{|c|}{ GEI $(n=16)$} \\
\hline $\mathrm{Me}$ & 134 & $88 *$ & 84 & 85 \\
\hline Q1 & 83,5 & 68,5 & 81,5 & 83 \\
\hline Q3 & 169 & 130,5 & 89 & 95 \\
\hline
\end{tabular}

* $p<0,05$ em relação ao pré-treinamento, teste de Wilcoxon

Além das medidas antropométricas e bioquímicas, avaliaram-se os efeitos fisiológicos do trabalho físico antes e depois do treinamento aeróbio contínuo (GEC) e anaeróbio intermitente (GEI).

Os valores médios do LAn (limiar anaeróbio) do GEC e GEI estão representados na tabela 7. Não foi encontrada diferença significativa nos valores iniciais entre os grupos (teste de Mann-Whitney). Após o treinamento físico, houve aumento significativo na velocidade do LAn para ambos os grupos (GEC, $p=0,001 ; G E I, p=$ $0,002)$. A resposta da freqüência cardíaca (FC) diminuiu para ambos os grupos com valor estatístico somente no GEI ( $p=0,004)$.

TABELA 7

Limiar anaeróbio (LAn) e freqüência cardíaca referente ao limiar anaeróbio $\left(\mathrm{FC}_{\mathrm{lan}}\right)$ dos grupos aeróbio contínuo (GEC) e anaeróbio intermitente (GEI), pré e pós-treinamento. Valores expressos como mediana (Me) e amplitude semiquartil (01 e Q3)

\begin{tabular}{|c|c|c|c|c|}
\hline \multirow[t]{2}{*}{ Grupo } & \multicolumn{2}{|c|}{ LAn (km/h) } & \multicolumn{2}{|c|}{$\mathrm{FC}_{\text {lan }}$ (bpm) } \\
\hline & Pré & Pós & Pré & Pós \\
\hline \multicolumn{5}{|c|}{ GEC ( $n=13)$} \\
\hline $\mathrm{Me}$ & 8 & $9,3^{*}$ & 179,1 & 174,6 \\
\hline Q1 & 7,6 & 8,1 & 175 & 168,6 \\
\hline Q3 & 8,3 & 9,7 & 181 & 177,5 \\
\hline \multicolumn{5}{|c|}{$\mathrm{GEI}(\mathrm{n}=16)$} \\
\hline $\mathrm{Me}$ & 7,35 & $7,65^{*}$ & 180,3 & $177^{*}$ \\
\hline Q1 & 6,59 & 7,56 & 176,95 & 167,5 \\
\hline Q3 & 7,8 & 8,68 & 193,1 & 180,5 \\
\hline
\end{tabular}

* $p<0,05$ em relação ao pré-treinamento, teste de Wilcoxon

Os valores de consumo máximo de oxigênio medido em litros por minuto (absoluto) e divididos por quilograma de peso corporal (relativo) são apresentados na tabela 8. Não houve diferença estatística dos valores iniciais entre os grupos. Avaliando a evolução desse parâmetro dentro dos grupos, tanto o GEC e GEl apresentaram aumento com diferença estatística no consumo máximo de oxigênio absoluto e relativo quando comparados os períodos inicial e final (GEC, $p=0,017$ e 0,028; GEl, $p=0,001$ e 0,001, respectivamente).
TABELA 8

Consumo máximo de oxigênio: valores absolutos $\left(\mathrm{V}_{2} \mathrm{absoluto}_{2}\right)$ e relativos $\left(\mathrm{VO}_{2}\right.$ relativo) dos grupos aeróbio contínuo (GEC) e anaeróbio intermitente (GEI), nos períodos pré e pós-treinamento. Valores expressos como mediana (Me) e amplitude semiquartil (01 e Q3)

\begin{tabular}{|c|c|c|c|c|}
\hline \multirow[t]{2}{*}{ Grupo } & \multicolumn{2}{|c|}{$\dot{\mathrm{VO}}_{2}$ absoluto (I/min) } & \multicolumn{2}{|c|}{$\dot{\mathrm{V}} \mathrm{O}_{2}$ relativo $(\mathrm{ml} / \mathrm{min} / \mathrm{kg})$} \\
\hline & Pré & Pós & Pré & Pós \\
\hline \multicolumn{5}{|c|}{ GEC ( $n=13)$} \\
\hline $\mathrm{Me}$ & 2,84 & $3,47^{*}$ & 35,25 & $46,75^{*}$ \\
\hline Q1 & 2,42 & 3,08 & 33 & 39,8 \\
\hline Q3 & 3,33 & 4,46 & 36,97 & 47,24 \\
\hline \multicolumn{5}{|c|}{ GEI $(n=16)$} \\
\hline $\mathrm{Me}$ & 3,03 & $3,56^{*}$ & 35,93 & $45,15^{*}$ \\
\hline Q1 & 1,78 & 3,4 & 32,95 & 39,19 \\
\hline O3 & 3,27 & 3,92 & 40,88 & 50,23 \\
\hline
\end{tabular}

* $p<0,05$ em relação ao pré-treinamento, teste de Wilcoxon

\section{DISCUSSÃO}

Muitos estudos têm demonstrado efeito simultâneo da dieta e da atividade física para prevenção e tratamento da obesidade. Contudo, os mecanismos de ação e otimização da dieta e dos exercícios necessitam ser melhor esclarecidos. Algumas das divergências encontradas nos resultados relatados quanto ao efeito da dieta e do exercício físico na massa corporal, na composição corporal e na taxa de metabolismo basal de pessoas obesas podem ser atribuídas a vários fatores, como a intensidade do exercício, magnitude da restrição calórica, quantidade e distribuição da gordura corporal inicial, combinações variadas de dieta e exercício utilizando diferentes protocolos 21,22$)$.

Sothern et al. ${ }^{(23)}$ utilizaram um programa de treinamento físico de moderada intensidade e progressivo, definido como 45 a $55 \%$ do $\dot{\mathrm{V}} \mathrm{O}_{2}$ max, juntamente com dieta e mudança de hábitos alimentares com avaliações antropométricas realizadas com 10 e 30 semanas em crianças obesas. As crianças diminuíram o IMC e a percentagem de gordura também, mas não houve mudança na massa magra corporal. Dos 11 sujeitos avaliados, oito apresentaram respostas positivas ao controle da massa corporal, ou seja, aproximadamente $72 \%$ diminuíram a massa corporal total e $90 \%$ diminuíram o IMC.

Podemos, neste nosso estudo, confirmar essas modificações ocorridas nestes mesmos parâmetros. Nos exercícios aeróbios, a massa corporal total diminuiu 1,5\% e nos exercícios anaeróbios, $3,7 \%$. Isso determinou a diminuição do IMC em 4,5\% no GEC e em $7,6 \%$ no GEI.

Foi observado nos resultados pós-exercício aumento significante estatisticamente na massa magra e diminuição na massa gorda, tanto no GEC quanto no GEl. Esses resultados estão de acordo com Krotkiewiski(24) e outros autores citados a seguir, mostrando que a atividade física atua na preservação da massa magra e na perda de gordura.

Quando o exercício é comparado diretamente com a dieta, ou quando o exercício é associado com a dieta e comparado com a restrição calórica, a atividade física resulta na preservação da massa magra e diminuição de gordura, sendo que durante um programa de redução da massa corporal somente com dieta sem exercício o resultado é perda de menos gordura e mais musculatura(25). Tanto nos estudos com animais realizados por Oscai (1971) apud Powers e Howley(26), quanto nos humanos ${ }^{(27)}$, utilizando a restrição dietética isoladamente, a perda de massa corporal magra pode ser igual a 30-40\% do peso perdido. O exercício associado à dieta acarreta menor perda de massa corporal magra e, proporcionalmente, maior perda de gordura ${ }^{(26,28)}$. Além disso, a mobilização preferencial da gordura do tecido adiposo visceral resulta em melhoria de sua distribuição corporal e do perfil do fator de risco(29) 
Fernandez ${ }^{(30)}$, que avaliou 28 adolescentes obesos divididos em grupo aeróbio, anaeróbio e controle, concluiu que os exercícios anaeróbios também são eficientes para a diminuição de gordura.

Pudemos confirmar estes dados com os resultados do nosso presente estudo, verificando a eficiência do exercício anaeróbio para a perda da massa corporal, bem como para a melhora cardiorrespiratória, medidas bioquímicas e na composição corporal.

Santarém ${ }^{(31)}$ e Ceddia(32) também afirmam que os exercícios anaeróbios podem promover alta mobilização de ácidos graxos livres e, conseqüentemente, controle sobre os níveis teciduais de gordura, uma vez que a manutenção e/ou aumento da massa magra através de exercícios resistidos (de força) tende a manter o metabolismo basal elevado por várias horas após os esforços anaeróbios, pelo fato de o tecido muscular se manter metabolicamente mais ativo mesmo em estado de repouso.

Nas medidas bioquímicas avaliadas neste estudo, os níveis de HDL e LDL apresentaram diminuição no GEC, com diferença estatística. No GEI o HDL também diminuiu estatisticamente, mas houve aumento do LDL sem diferença estatística, apesar de não ser fora dos parâmetros previsto para a idade, bem como a diminuição do HDL para os dois grupos. As respostas de triglicérides (TG) aumentaram estatisticamente no GEC e diminuíram no GEl. A glicemia aumentou estatisticamente no GEC e no GEl não houve alteração significativa. Os níveis de colesterol mostraram diminuição nos dois grupos, com diferença significativa apenas no GEC.

O exercício exerce pequeno efeito sobre o nível de LDL-colesterol quando são levados em conta fatores como composição corporal e ingestão dietética de lipídios e colestero|(29).

Silva et al. ${ }^{(34)}$, em seu estudo com ratos, verificaram o efeito dos exercícios aeróbios e anaeróbios sobre a gordura sérica e tecidual de ratos alimentados com dieta hiperlipídica. Concluíram que o treinamento anaeróbio pode constituir estímulo tão ou mais eficiente quanto o aeróbio na redução dos efeitos negativos vindos do excesso de gordura da dieta, tanto em relação a alterações nos lipídios séricos quanto nos teciduais.

Curiosamente, Reaven et al., apud Macauley et al.(35), verificaram que o HDL estava aumentado em homens e mulheres idosos e ativos (50 a 89 anos de idade), havendo uma relação dose-resposta nos indivíduos do sexo masculino, enquanto os níveis de HDL estavam levemente mais baixos nas mulheres que exercitavam atividade intensa.

No presente estudo, houve também diminuição nos níveis séricos de HDL, nos dois grupos (GEC e GEl), apesar desses valores estarem dentro dos parâmetros considerados normais.

Em relação à avaliação da capacidade física dos adolescentes obesos, no nosso estudo, os resultados das respostas do consumo máximo de oxigênio ( $\mathrm{V}_{2} \max$ ) e de LAn (limiar anaeróbio) aumentaram estatisticamente no GEC e GEI, valores esses suficientes para que eles pudessem melhorar sua capacidade de trabalho.

Quanto ao fato de as respostas de $\dot{\mathrm{VO}}_{2}$ max apresentarem diferença estatística nos dois grupos referentes ao treinamento realizado, pode-se concluir que tanto o exercício aeróbio quanto o anaeróbio foram eficientes para a melhora na capacidade física dos adolescentes obesos, pois houve alteração positiva nesses resultados tanto no GEC (de 35,4 para $42,7 \mathrm{ml} . \mathrm{kg}^{-1}$. $\mathrm{min}^{-1}$ ) quanto no GEI (de 37 para $45 \mathrm{ml} \mathrm{kg}^{-1} \cdot \mathrm{min}^{-1}$ ).

Os valores referentes ao $\dot{\mathrm{VO}}_{2}$ max para os dois grupos obtidos no presente estudo foram semelhantes aos encontrados por Fernandez ${ }^{(30)}$, que, avaliando 28 adolescentes, verificou o $\mathrm{VO}_{2}$ max relativo de $36,50 \pm 6,33 \mathrm{ml} \cdot \mathrm{kg}^{-1} \cdot \mathrm{min}^{-1} ; 32,08 \pm 2,61 ; 29,91 \pm$ $6,49 \mathrm{ml} \cdot \mathrm{kg}^{-1} \cdot \mathrm{min}^{-1}$, para os grupos aeróbio, anaeróbio e controle, respectivamente. Esses resultados revelam que os dois tipos de treinamento utilizados aumentaram o consumo máximo de oxigênio, quando comparados com o grupo que não realizou atividade.

Estudo semelhante ao nosso foi realizado por Woitge et al.(36), que treinaram dois grupos de jovens de 20 a 29 anos durante oito semanas, utilizando treinamento aeróbio (40 a 60 minutos de dura- ção com intensidade 60 a $85 \% \dot{V}_{2}$ max) e anaeróbio ('tiros' de corrida com 90 a 100\% da velocidade máxima de cada voluntário). Eles concluíram que os dois tipos de treinamento aumentaram a capacidade aeróbia (determinada pelo limiar anaeróbio) em 10\% após oito semanas de intervenção. Esses resultados foram semeIhantes aos encontrados no nosso protocolo, aumentando no GEC $=6,5 \%$ e no $\mathrm{GEI}=8,1 \%$ do LAn.

Diante dos resultados obtidos em cada grupo, podemos concluir que o exercício físico aeróbio promoveu redução da massa corporal total acompanhada de melhora na composição corporal, mudança no perfil lipídico e melhora também na capacidade física. O exercício anaeróbio se comportou de forma semelhante diante das medidas analisadas, sendo melhor a resposta da capacidade física com relação à freqüência cardíaca.

A orientação nutricional foi importante e útil para produzir mudanças no padrão e hábitos alimentares dos adolescentes e suas famílias. Apesar de a adesão à orientação nutricional não ter sido monitorizada com rigor, os dados encontrados evidenciaram que eles tiveram mudança de hábitos, pois só o exercício físico provavelmente não provocaria tais resultados.

Essa orientação, baseada na reeducação alimentar, teve reuniões com periodicidade semanal para os adolescentes e mensal para os pais. Com propósito educativo e informativo, procurou incentivá-los na mudança de hábitos e comportamentos, buscando uma alimentação mais saudável e equilibrada. Nas reuniões com os pais em conjunto com seus filhos, pudemos ouvir declarações em relação a essas mudanças de hábitos, como por exemplo:

. " estou conseguindo mudar os meus hábitos alimentares, antes não comia fruta, hoje tenho comido sempre".

.."tenho me sentido mais motivado em mudar os maus hábitos, pois tenho percebido resultados positivos em minha vida, já não tenho mais vergonha de praticar esportes diante dos outros".

..."antes mal conseguia caminhar e já me sentia cansado, hoje já consigo correr, praticar esportes na minha escola e tenho prazer por isso".

Diante de todos os resultados apresentados, podemos sugerir que os dois tipos de exercícios, aeróbios e anaeróbios, associados à orientação alimentar, foram satisfatórios em promover mudanças corporais e comportamentais importantes, devendo ser adotados em programas de atividade física auxiliando o combate à obesidade na adolescência e prevenindo futuras complicações na fase adulta.

Apesar dessas considerações, estamos cientes das limitações deste estudo em relação ao tamanho da amostra e por não haver grupo controle sem qualquer intervenção. Isso não ocorreu por dificuldades técnicas e disponibilidade de sujeitos para a pesquisa, que envolveu um protocolo árduo e denso. No entanto, temos certeza de que se esses aspectos fossem preenchidos, resultados muito mais consistentes seriam obtidos, enriquecendo a discussão e fortalecendo as conclusões. Deixamos aqui propostas para novos estudos com este protocolo, com grupo controle e grupo com exercícios associados (anaeróbio e aeróbio).

Todos os autores declararam não haver qualquer potencial conflito de interesses referente a este artigo.

\section{REFERÊNCIAS}

1. Pollock ML, Wilmore JH. Medicina e esporte. In: Exercício na saúde e na doença: avaliação e prescrição para prevenção e reabilitação. $2^{\underline{a}}$ ed. Rio de Janeiro: 1993;125-7.

2. Perri MG, Nezu AM, Viegener BJ. Obesity: definition, prevalence and consequences. In: Improving the long-term management of obesity: theory, research, and clinical guidelines. John Wiley \& Sons, 1992;3-24.

3. World Health Organization (WHO). Adolescents. In: Physical status: the use and interpretation of anthropometry. Geneva: WHO, 1995;263-311.

4. Lamounier AJ. Situação da obesidade na adolescência no Brasil. In: Dutra de Oliveira JE, Lamounier AJ, Berezovsky MW, Portella Jr OA (coords.). Obesidade 
e anemia carencial na adolescência: simpósio. São Paulo: Instituto Danone, 2000;15-31.

5. Montoanelli G, Bittencourt VB, Oenteado RZ, Pereira IMTB, Alvarez, MCA. Educação nutricional: uma resposta ao problema da obesidade em adolescentes. Revista Brasileira de Desenvolvimento Humano, São Paulo, 1997;7:85-93.

6. Schutz Y. Macronutrients and energy balance in obesity. Metabolism 1995:44: S7-11.

7. Dietz WH, Gortmaker SL. Do we fatten our children at the television set? Obesity and television viewing in children and adolescents. Paediatrics 1985;75:80512.

8. Fisberg M. Obesidade na infância e adolescência. São Paulo: Fundação Byk, 1995.

9. Bouchard C, Tremblay A. Genetic effects in human energy expenditure components. Int J Obes 1990;14:S49-58.

10. United States Of America. Public Health Service. Department of Health and Human Services. Promoting health/prevention disease: objectives for the $\mathrm{Na}$ tion. Washington: U.S. Government Printing Office, 1980.

11. Ruiz AT, Ruiz JVT. Aspects practices del treatment de I'obesity infantil. Apunts 1993;30:117-34.

12. Negrão CE, Trombetta IC, Tinucci T, Forjaz CLM. O papel do sedentarismo na obesidade. Revista Brasileira Hipertensão 2000;2:149-55.

13. Axelrud E, Gleiser D, Fischmann J. Obesidade na adolescência: uma abordagem para pais, educadores e profissionais da saúde. Porto Alegre: Mercado Aberto, 1999;15-20.

14. Viuniski N. Obesidade infantil: um guia prático para profissionais da saúde. Rio de Janeiro: EPUB, 1999.

15. Damaso AR, Teixeira LR, Curl CMON. Atividades motoras na obesidade. In: Fisberg M. Obesidade na infância e adolescência. São Paulo: Fundo Editorial Byk, 1995;91-9.

16. Must A, Dallai GE, Dietz WH. Reference data obesity: $85^{\text {th }}$ and $95^{\text {th }}$ percentiles of body mass index (wt/ht2) and triceps skinfold thickness. Am J Clin Nutr 1991; 53:839-46.

17. Garrow JS, Webster J. Quetelet's index $(w / H)$ as a measure of fatness. Int J Obes 1985;9:147-53.

18. Tubino MJG. Metodologia científica do treinamento desportivo. $3^{\underline{a}}$ ed. São PauIo: Ibrasa, 1983.

19. Moreira SB. Equacionando o treinamento - A matemática das provas longas. Rio de Janeiro: Shape, 1996.

20. Sharkey BJ. Condicionamento físico e saúde. 4aㅡ ed. São Paulo: Artemed, 1998.

21. Whatley JE, PoehIman ET. Obesity and exercise. In: Blackburn GL, Kandres BS, editors. Obesity: path physiology and treatment. New York: Chapman \& Hall, 1994.
22. Barbosa AR, Santarém JM, Jacob Filho W, Meirelles ES, Marucci JM. Comparison of body fat using anthropometry bioelectrical impedance and DEXA in elderly women. Arch Latinoam Nutr 2001;51:49-56.

23. Sothern MS, Hunter S, Suskind RM, Brown R, Udall JN, Blecker U. Motivating the obese child to move: the role of structured exercise in pediatric weight management. South Med J 1999;92:577-84.

24. Krotkiewski M. Role of muscle morphology in the development of insulin resistance and metabolic syndrome. Presse Med 1994;23:1393-9.

25. Baron R. Understanding obesity and weight loss.1995. Disponível em: http:// wwwmed.stanford.edu/school/DGIM/Teaching/+Modules/obesity.html. Acesso em: 28 ago.1997.

26. Powers SK, Howley ET. Fisiologia do exercício. Teoria e aplicação ao condicionamento e ao desempenho. 3a ed. São Paulo: Manole, 2000.

27. Durnin JVGA. Possible interaction between physical activity, body composition, and obesity in man. In: Bray G. Recent advances in obesity research. $2^{\mathrm{a}}$ ed. Westport: Technomic, 1978.

28. Schwingshandl J, Sudi K, Eibl B, Wallner S, Borkenstein M. Effect of an individualized training programmed during weight reduction on body composition: a randomized trial. Arch Dis Child 1999;81:426-8.

29. Ross R, Rissanen J. Mobilization of visceral and subcutaneous adipose tissue in response to energy restriction and exercise. Am J Clin Nutr 1994;60:695-703.

30. Fernandez AC. Influência do exercício aeróbio e anaeróbio na composição corporal de adolescentes obesos, 2001. 136p. Dissertação (Mestrado) - Escola Paulista de Medicina, Universidade Federal de São Paulo, São Paulo.

31. Santarém JM. Musculação e qualidade de vida. Rev Bras Med Esporte 1996;2: $11-4$.

32. Ceddia RB. Gordura corporal, exercício e emagrecimento. Reviews Sprint Magazine 1998;1:10-20.

33. Wood P, Stefanick ML, Willian PT, Haskell WL. The effects on plasma lipoproteins of a prudent weight-reducing diet, with or without exercise, in overweight men and women. N Engl J Med 1991;325:7:461-6.

34. Silva PM, Marcondes MCCG, Mello AR. Exercícios aeróbio e anaeróbio: efeitos sobre a gordura sérica e tecidual de ratos alimentados com dieta hiperlipídica. Revista Atividade Física e Saúde 1999;4:43-56.

35. Macauley D, Mccrum EE, Stott G, Evans AE, Duly E, Trinick T, et al. Physical activity, lipids, apoliproteins, and Lp(a) in the Northern Ireland Health and Activity Survey. Med Sci Sports Exerc 1996;28:720-36.

36. Woitge BF, Henning W, Suttner S, Farahmand I, Martin C. Changes in bone turnover induced by aerobic and anaerobic exercise in young males. J Bone Min Met 1998;13:1797-804. 